\title{
The diagnostic and prognostic role of cytokines in colon cancer
}

This article was published in the following Dove Press journal:

Gastrointestinal Cancer:Targets and Therapy

2I April 20I I

Number of times this article has been viewed

\section{Tiha M Long \\ Jean-Pierre Raufman}

Department of Medicine, Division of Gastroenterology and Hepatology, University of Maryland School of Medicine, Baltimore, MD, USA
Correspondence: Jean-Pierre Raufman Division of Gastroenterology and Hepatology, University of Maryland School of Medicine

22 South Greene Street, N3W62

Baltimore, MD 2I 20I, USA

$\mathrm{Tel}+\mathrm{I} 4103288728$

Fax + I 410328 83I5

Email jraufman@medicine.umaryland.edu
Abstract: In the United States, colorectal cancer (CRC) is the third most common cancer diagnosis in both men and women. Many patients are diagnosed with advanced stage CRC which has a high rate of mortality compared to localized CRC. Developing novel diagnostic tools is important for early detection to decrease both morbidity and mortality. Currently, colonoscopy is the most common screening test, though its effectiveness in reducing mortality remains uncertain and it is costly, invasive and carries risk. Stool-based tests such as fecal occult blood test and fecal immunochemical test for hemoglobin are relatively inexpensive, cause no discomfort and have no risk but lack sufficient specificity or sensitivity. Stool DNA tests may be more sensitive, but are not cost-effective and have not been sufficiently evaluated in population-based studies. To improve diagnostic efficacy, it is necessary to develop tests that limit patient discomfort and are cost-effective while providing adequate sensitivity and specificity. Elucidating serum biomarker profiles for various stages of CRC would provide a platform from which to develop enzyme-linked immunosorbent and multiplex bead array assays for clinical use. Both inflammation-induced and sporadic CRC trigger immune responses that are mediated by cytokine production. Many inflammatory serum proteins have been analyzed for their ability to predict cancer. Patient cohort studies have detected differential expression of inflammatory serum biomarkers in healthy controls compared to persons with various stages of CRC. Moreover, proteomics and animal research have uncovered additional serum factors implicated in CRC. Analysis of known differentially-expressed biomarkers along with further study of potential biomarkers may lead to clinically useful serum biomarker profiles for CRC.

Keywords: cytokine, multiplex bead array, inflammatory bowel disease, colitis-associated cancer

\section{Introduction}

In the United States colorectal cancer (CRC) is the third most common cancer diagnosis in both men and women, and the second most common cause of cancer death in men and women combined. Approximately $25 \%$ of CRC is familial and $\sim 1 \%$ develops in individuals with chronic inflammatory bowel disease (IBD). ${ }^{1}$ IBD, an immune-mediated disease, affects the gastrointestinal tract primarily and is classified broadly as either Crohn's disease (CD) or ulcerative colitis (UC). Estimates from 2004 data suggest that approximately 1.4 million people in the US have IBD. UC and CD increase risk for CRC development but the causal relationship between UC and CRC is better established than that between CD and CRC. ${ }^{2,3}$ Overall, individuals with UC have a $>10$-fold increased risk of developing CRC. ${ }^{4}$ Other risk factors for colon cancer include advanced age, cigarette smoking, obesity and diets rich in meat and fat. ${ }^{5}$ 
CRC rates have declined since 1998. This decline is most likely multi-factorial, including increased screening and polyp removal. Nonetheless, nearly 50,000 people died from CRC in 2008. Early detection is important to reduce CRC mortality; 5-year survival for localized CRC is $\sim 90 \%$, whereas regional spread reduces survival to $\sim 68 \%$ and for metastatic disease, 5 -year survival drops to $\sim 11 \%{ }^{6}{ }^{6}$ From 1996-2004, 60\% of patients with CRC had advanced disease. ${ }^{6}$ These statistics highlight the need to develop novel diagnostic tools to detect the disease at early stages, thereby increasing the likelihood of survival.

Colorectal cancer screening reduces mortality by detecting and removing pre-cancerous adenomas and by detecting cancers at an earlier, more curable, stage. Colonoscopy and sigmoidoscopy are current methods to identify and remove pre-cancerous adenomas. Non-invasive tests that detect but cannot remove CRC include fecal occult blood tests (FOBT), fecal immunochemical test for hemoglobin (FIT), stool DNA (sDNA) and computerized tomographic (CT) colonography. Each of these screening tests has limited sensitivity and specificity.

In the United States, colonoscopy has become the primary screening test. This method is thought to decrease overall CRC mortality through early detection but the effectiveness of colonoscopy remains unproven. Although there is general agreement that screening colonoscopy reduces overall CRC mortality, recent studies suggest that colonoscopy may not alter mortality from right-sided CRC (ascending colon and cecum). ${ }^{7}$ Moreover, colonoscopy is time-consuming, costly, invasive, and carries sedation- and instrumentation-related risks (eg, respiratory arrest, perforation and bleeding). Additionally, in average-risk populations colonoscopy is costeffective only if performed every 10 years. Sigmoidoscopy can detect polyps in the distal colon but may miss advanced $\mathrm{CRC}$ in the proximal colon. Also, since it is commonly performed without sedation sigmoidoscopy is generally uncomfortable and is not cost-effective in most practices.

In comparison to endoscopy, stool-based tests are relatively inexpensive, cause no discomfort and have no risk. These methods can diagnose cancer but are less likely to detect adenomas. FOBT lacks sufficient specificity or sensitivity, thus identifying only approximately $50 \%$ of patients with CRC. FIT, a commercially-available test, measures hemoglobin levels in fecal material. Direct comparisons of FIT and FOBT are not available, and false-positive results with FIT are possible. ${ }^{8}$ Stool-based DNA (sDNA) tests that examine excreted cellular DNA for specific genetic mutations have a higher specificity than FOBT in identifying early cancers.
Though sDNA may detect CRC more often than FOBT, the test is less cost-effective and requires the collection of an entire stool specimen followed by careful preservation and processing to maintain DNA stability. These tests use polymerase chain reaction (PCR) to detect multiple gene mutations including point mutations in K-ras, APC and p53, plus markers of DNA integrity and microsatellite instability. ${ }^{9}$ Newer versions of this method are currently being evaluated and population-based studies are required to determine if sDNA tests perform better than FOBT and FIT.

These observations identify a great need for diagnostic and prognostic tests for CRC that are cost-effective, noninvasive and practical for widespread application. Defining serum biomarker profiles that are sensitive and specific for chronic IBD and various stages of CRC including tumor initiation, promotion, invasion and metastasis could provide the basis to develop enzyme-linked immunosorbent (ELISA) and multiplex bead array assays for clinical use. These techniques use antibodies to measure protein concentrations. Multiplex bead arrays permit the detection of a large number of proteins in a small volume of serum. The initial requirement in the development of such biomarker panels is the detection of differentially expressed proteins in persons with colon neoplasia versus healthy controls, and differentially expressed proteins in IBD and various stages of colon cancer. A plethora of inflammatory proteins are involved in inflammation-induced neoplasia and in the innate immune response to tumor growth in sporadic CRC. ${ }^{1}$ Serum mediators of the immune response provide a source from which to identify differentially expressed factors. Animal studies have expanded knowledge regarding the role of inflammatory mediators in CRC. In addition, proteomics has emerged as a powerful technology for detection of differentially expressed proteins in samples and has been used to develop biomarker panels through analysis of patient serum samples and cell lines. With varying levels of success, studies have examined the concentration of cytokines and other serum proteins in patient cohorts. Patient cohort studies include groups that have average risk for CRC and higher risk groups such as patients with IBD or obesity, which promotes chronic systemic inflammation and is a risk factor for CRC. ${ }^{10}$ This review will critically evaluate recent research regarding CRC and the inflammatory response, patient cohort studies and emerging proteomics techniques for developing biomarker panels. Analysis of available data may lead to the development of serum biomarker profiles of diagnostic and prognostic value in diagnosing and monitoring the progression of colon neoplasia. 


\section{Inflammation-induced colon cancer}

A variety of factors contribute to the development of CRC including genetic abnormalities and chronic inflammation. Sporadic CRC (SCRC) arises from genetic instability whereas inflammation-induced $\mathrm{CRC}$ is initiated by chronic IBD. Though both UC and CD increase risk of CRC development the association of $\mathrm{CD}$ with $\mathrm{CRC}$ is moderate, whereas the elevated cancer risk in UC patients is welldefined. ${ }^{2}$ Sporadic and colitis-associated cancer (CAC) evolve through different pathways. SCRC progresses through an adenoma-carcinoma sequence, whereas CAC develops as a result of inflammation-induced dysplasia which can progress to carcinoma. In addition, there is a discrepancy in the genetic progression of SCRC and CAC. A key step in SCRC is the activation of $\mathrm{Wnt} / \beta$-catenin-signaling as a consequence of adenoma polyposis coli (APC) gene inactivation and nuclear translocation of $\beta$-catenin, a transcription factor that contributes to aberrant cell proliferation. Loss of APC is an initiating event in SCRC often followed by K-ras oncogene activation and p53 tumor suppressor gene inactivation. In contrast, p53 mutations occur in the pre-invasive stage of $\mathrm{CAC}$ and Src oncogene acivation is a common early event, whereas APC and K-ras mutations are less common and occur later. ${ }^{11}$ SCRC and CAC also differ in relationship to inflammation. Colitis-associated neoplasia is induced by the inflammatory response, whereas inflammation follows tumor development in SCRC. ${ }^{1}$

The link between inflammation and cancer is well established. ${ }^{12}$ Infiltrating immune cells and the production of cytokines and chemokines create a pro-tumorigenic microenvironment which contributes to cancer initiation and progression. CAC exemplifies this process. It is speculated that UC drives CAC formation by promoting DNA damage through sustained oxidative stress and increased IEC turnover due to the inflammatory microenvironment. Also, drugs that treat IBD, such as the NSAID 5-aminosalicylates (5-ASA), may inhibit the development of CRC. ${ }^{3}$

Pro-inflammatory cytokines and chemokines are compulsory factors in inflammation-induced cancers, including colitis-associated cancer (CAC). Cytokines modulate most aspects of inflammation including homeostasis, mobility and differentiation. By analyzing cytokine pathways involved in $\mathrm{UC}$ and $\mathrm{CAC}$ it is possible to gain a more comprehensive understanding of CAC tumorigenesis evolving from UC. Many cytokines including IL-1 $\beta$, IL-2, IL-4, IL-5, IL-6, TNF $\alpha$, IL-8, IL-10, IL-11, IL-12, IL-17, IL-18, IL-11, IL-22, IL-23, TGF- $\beta$ and IFN $\gamma$ dysregulation are implicated in UC and progression to CAC.,13-15 Some cytokines promote all stages of tumor development from initiation and promotion to invasion and metastasis, whereas others are stage-specific. In particular, increased levels of pro-inflammatory TNF $\alpha$, IL-17, IL-23, IFN $\gamma$ and IL-6 are implicated in tumorigenesis; several, such as TNF $\alpha$, IL-1 IL-6, IL-11, IL-22 and IL-23 are involved in tumor promotion. Due to its role in epithelialmesenchymal transition (EMT) TGF $\beta$ is active in promotion and invasion. IL-1, IL-6 and TNF $\alpha$ also play a role in tumor invasion and metastasis. Another marker of inflammation, C-reactive protein (CRP) is produced by liver cells in response to circulating IL-6. This acute phase protein is important for opsonization of pathogens and cellular debris. CRP is upregulated in many cancers including CRC and is broadly used for diagnosing inflammation and malignancy. ${ }^{16}$ In addition to promoting $\mathrm{CAC}$ development, cytokines are also found in the serum of patients with SCRC after tumor development and may influence prognosis. ${ }^{1}$

Changes in cytokine expression have been explored extensively in murine models of UC and CAC. One commonly used system is the dextran sulfate sodium/ azoxymethane (DSS/AOM) mouse model. This murine model mimics human disease by increasing intestinal epithelial permeability, thereby facilitating transmucosal infiltration of intestinal bacteria and carcinogens. Interruption of epithelial homeostasis leads to immune cell infiltration and an inflammatory response which mimics human UC. Treatment with the mutagen AOM increases tumor occurrence by promoting genomic instability in colonic epithelial cells. Treatment with DSS only is used to induce severe colitis, or can be cycled at low concentrations to mimic chronic inflammatory disease which causes CAC. DSS treatment was shown to reversibly induce the expression of various cytokines including IL-1 $\beta$, TNF $\alpha$, IFN $\gamma$, IL-6, IL-10, IL-12, and chemokines MIP-2 and KC. ${ }^{17}$ Several mouse models of UC and CAC involve deficient cytokine production or signaling as indicated by the use of transgenic mice to investigate UC and CAC. These models include: IL-2/ IL-2R ${ }^{-/-}$, IL-10 $0^{-/-}$and TNF-UTR mutants. Results from these models indicate that colitis develops concurrently with dysregulated inflammatory cytokine expression and/or decreased anti-inflammatory cytokine expression. Animal models may also overexpress cytokines, for example Il-17 transgenic mice and Stat-4 overexpressing mice are used in UC and CAC studies. On-going research continues to expand the number of known cytokine effectors in UC and CAC.

The prevalence of cytokines in CRC provides a plethora of serum protein markers to investigate. Continued research into the expression of cytokines and other serum biomarkers 
in CAC and SCRC may lead to the development of improved diagnostic and prognostic tools. By analyzing serum biomarkers in various stages of IBD and CAC progression, it may be possible to identify differential serum profiles for chronic inflammation or specific CAC stage. Furthermore, cytokines produced in response to SCRC may provide prognostic clues. In comparison to currently used CRC tests, serum analysis could offer a diagnostic and prognostic test with reduced invasiveness and increased patient comfort. Such screening methods may increase adherence to screening guidelines and detect early stage CRC thereby reducing overall morbidity.

\section{Cytokine function in animal models of UC and CAC}

Cytokines are small signaling proteins with diverse functions in inflammation. Cytokines control both innate and adaptive immunity and are involved in early stages of inflammation, such as immune cell migration and infiltration, and later stages of cell-mediated immunity, such as cytotoxic T cell activation and antibody production. Both innate and adaptive immune responses play a role in UC and CAC. The biological function of various cytokines influences the disease stage in which they are involved. For example, some cytokines are involved in the inflammatory response while others are involved in cell survival and differentiation. These differences in function determine if a cytokine is likely to be involved in IBD, colorectal carcinogenesis or metastasis.

Immune signaling has been studied extensively in CAC pathogenesis, including IL-1 and IL-18 activation by the nucleotide-binding domain, leucine-rich-repeat (NLR) inflammasome. This early immune response is due to loss of intestinal epithelial homeostasis and bacterial infiltration. ${ }^{18}$ Activation of IL-1 and IL-18 can induce downstream activation of additional cytokines and chemokines that promote inflammation. Tumor necrosis factor alpha $(\mathrm{TNF} \alpha)$ is a pro-inflammatory cytokine implicated in colon tumorigenesis and extensively investigated in CRC. TNF $\alpha$ signaling leads to activation of the transcription factor $\mathrm{NF}-\kappa \mathrm{B}$ which can promote aberrant cell differentiation and proliferation. Cell-mediated immunity is also important in maintaining chronic inflammatory diseases such as UC. IL-2, IL-10 and IL-12 manipulate cell-mediated adaptive immunity. Moreover, chemokines have been shown to play a role in UC and CAC by controlling immune cell infiltration and cell mobility. Animal models can be used to detect differentially expressed serum biomarkers in IBD and colon cancer. Recent research has expanded knowledge of the function of cytokines in UC and CAC.

\section{A protective role for NLR inflammasomes and IL-I signaling in CAC development}

NLR inflammasomes are multiprotein complexes formed in response to cell stress or infection. Complex formation leads to activation of caspase- 1 followed by cleavage of propeptide forms of IL-1 $\beta$ and IL-18 causing activation of these inflammatory cytokines. IL- $1 \beta$ and IL-18 are the primary stimulators of innate immunity and lead the response against pathogenic infection. Dysregulation of the NLR inflammasome pathway results in inability to process IL-1 $\beta$ and IL-18 and is associated with various disorders from chronic infection and auto-immunity to cancer. Defects in IL-1 $\beta$ are associated with increased risk of cancer including those of the gastrointestinal tract. ${ }^{19}$

Recent research supports a protective role for IL-1 $\beta$ in CRC. Specifically, the NLR inflammasome inhibits CAC development. In a mouse model of CAC, knocking out components of the inflammasome attenuated colon carcinogenesis. ${ }^{20}$ Compared to wild-type (WT) controls, mice lacking the inflammasome adaptor protein ASC/PYCARD or caspase-1 that were treated with dextran sulfate sodium (DSS) and azoxymethane (AOM) had increased disease severity and tumor burden. The investigators used ELISA to demonstrate reduced IL-1 $\beta$ and IL-18 production in the colon of DSS/AOM-treated inflammasome-deficient compared to control mice. To determine if NLR proteins modulate disease severity, NLRP3 ${ }^{-/}$and $\mathrm{NLRC}^{-/-}$mice were treated with DSS/AOM. NLRP3 ${ }^{-/-}$mice displayed more severe disease whereas $\mathrm{NLRC4}^{--}$mice did not have altered pathology. Data indicate that the NLRP3 inflammasome is involved in ASC/PYCARD binding and caspase-1 processing, though redundant pathways are likely to exist. Also, the NLRC4 inflammasome does not appear to be involved in disease susceptibility. NLRP3 $3^{-/}$mice were used to investigate which cell subsets were effectors of this phenotype. Following bone marrow transplantation using $\mathrm{NLRP}^{-/}$versus WT bone marrow, it was determined that hematopoietic cells deficient in NLRP3 inflammasome function contributed more to disease severity than epithelial, tumor or stromal cells. Overall, these data indicate that loss of NLR inflammasome function, particularly by immune cells, increases UC severity and progression to CAC. Also, in a DSS/AOM mouse model activated IL-1B and IL-18 attenuated UC and CAC. ${ }^{20,21}$ 
Other recent experimentation argues against a protective role for IL-1 signaling in colon tumorigenesis. Single immunoglobulin IL-1 receptor-related (SIGIRR) molecule is an inhibitor of the IL-1 and Toll-like receptor. Investigators crossed Apc ${ }^{\mathrm{min} /+}$ mice with SIGIRR knockout mice to determine the effects of SIGIRR in this mouse model of colon cancer. ${ }^{22}$ Due to loss of herterozygosity (LOH) of APC, $\mathrm{Apc}^{\mathrm{min} /+}$ mice primarily develop small intestine tumors. $\mathrm{Apc}^{\mathrm{min} /+} / \mathrm{Sigirr}^{-/-}$mice had increased carcinogenesis compared to $\mathrm{Apc}^{\mathrm{min} /+} / \mathrm{Sigirr}^{+/+}$mice. Increased tumor burden in $\mathrm{Apc}^{\mathrm{min} / /} / \mathrm{Sigirr}^{-/}$mice was attributed to increased activation of the Akt-mTOR pathway and increased $\mathrm{LOH}$ of the APC gene. Hyper-activation of Akt-mTOR increased following IL-1 or Toll-like receptor agonist treatment using either IL-1 or LPS. These results indicate a tumor suppressor role for the IL-1 and Toll-receptor inhibitor SIGIRR, and a tumor enhancing role for IL-1 activation of IL-1 receptor. These conflicting data may result from the use of different mouse models. The $\mathrm{Apc}^{\mathrm{min} /+}$ mouse is a model of sporadic colon cancer and not inflammation-induced cancer. Also, in addition to IL-1R ligand signaling SIGIRR protein inhibits Toll-like receptor activation. Interpretation of these results is difficult due to the dual function of this receptor and may not definitively address the role of IL-1 in colon carcinogenesis.

In addition to investigating IL-1 signaling in colon cancer, recent research has further investigated the effects of the inflammasome-processed cytokine, IL-18. One group investigated the effects of IL-1 $\beta$ and IL-18 on CAC using mice with inflammasome deficiencies including inflammasome adaptor protein knockout $\mathrm{Asc}^{-/}$, caspase-1 $1^{-/-}$and dominant negative caspase-1 inflammasome regulator: caspase-12-/ mice treated with DSS or DSS/AOM. With 5-day DSS-only treatment, caspase $-1^{-/-}$mice did not survive to day 9 and had drastically reduced body weight by day 5 , whereas caspase- $12^{-/-}$mice did not have reduced body weight, and $\mathrm{Asc}^{-/-}$mice had an intermediate phenotype. An increased presence of infiltrating bacteria was detected in the lamina propria of caspase- $1^{-/-}$mice which indicates that caspase-1 is necessary to maintain epithelial integrity and prevent bacterial invasion. To determine which cytokines were responsible for the severe caspase- $1^{-/-}$phenotype, WT mice were treated with DSS and IL-1 $\beta$ and IL-18 levels were measured. Interestingly, IL-18 serum levels were highly induced whereas IL-1 $\beta$ levels were minimally increased. The investigators hypothesized that treating caspase- $1^{-/-}$mice with IL-18 would rescue the phenotype. Indeed, administration of IL-18 following DSS treatment attenuated severe weight loss and bacterial invasion. To investigate recurring colitis and carcinogenesis, caspase- $12^{-/-}$mice were subjected to cycles of DSS and DSS/AOM treatment. Mice lacking the inflammasome negative regulator had increased resistance to chronic colitis. Strikingly, caspase- $12^{-/-}$mice had an increased tumor burden compared to WT mice. Overall, these data indicate a protective role for IL- $1 \beta$ in chronic colitis, but a deleterious role in colon carcinogenesis, and a protective role for IL-18 against UC. ${ }^{23}$

Another report both supports and refutes previous data. In this study, using a DSS/AOM model the authors first established that $\mathrm{Myd} 88^{-/-}$mice have increased colitis and colon cancer. Because Myd88 serves as an adapter protein for several IL-1 family receptors, the investigators treated IL-1R1 $1^{-/}$, IL-18R $\mathrm{R}^{-/}$, and IL-18 ${ }^{-/-}$mice with DSS/AOM to determine which pathway is altered by Myd88 deficiency to promote carcinogenesis. The protective effect of IL-18 was validated because, compared to DSS/AOM-treated WT mice, IL-1 $18^{-/-}$and IL-18R $\mathrm{R}^{-/}$mice were more susceptible to colitis and neoplasia. Disease in IL $18^{-/-}$mice was attenuated compared to MyD88 $8^{-/-}$mice, but further analysis showed similarities in certain cytokine levels, specifically increased IL- 6 and IL-11 and decreased IFN $\gamma$, thus indicating that in MyD88 $8^{-/-}$and IL-18 $8^{-/-}$mice increased disease severity results from overlapping pathways. This same study argues against the ability of IL-1B signaling to affect disease severity because in DSS/AOM-treated IL-1 $\mathrm{R}^{-/-}$mice there was no change in colitis or neoplasia. ${ }^{24}$

\section{TNF $\alpha / N F-\kappa B$ signaling promotes CAC}

Tumor necrosis factor-alpha $(\mathrm{TNF} \alpha)$ is a pro-inflammatory cytokine that binds TNFR and activates downstream signaling and activation of the transcription factor NF- $\mathrm{KB}$. Nuclear localization of NF- $\kappa \mathrm{B}$ promotes expression of additional pro-inflammatory cytokines. Depending on disease type and stage, TNF $\alpha$ and NF- $\mathrm{KB}$ have roles as both tumor suppressors and tumor promoters. Although stimulation of the $\mathrm{TNF} \alpha / \mathrm{NF}-\kappa \mathrm{B}$ pathway has anti-proliferative effects, $\mathrm{NF}-\kappa \mathrm{B}$ activation results in the transcription of a variety of genes including inflammatory cytokines and adhesion molecules that may increase the risk of inflammation-induced cancer and metastasis. Several studies showed that activation of NF- $\mathrm{\kappa B}$ promotes colitis-associated carcinogenesis.

Expression of TNF $\alpha$ in the colon was shown to increase throughout the process of colon carcinogenesis. To test the effects of inhibiting TNF $\alpha$ signaling, a DSS/AOM 
experiment was carried out using TNF receptor-deficient (TNFRp55 $5^{--}$) mice. Blocking TNF $\alpha$ signaling reduced colitis and tumor formation. ${ }^{25}$ Bone marrow transplantation experiments support a role for TNF $\alpha$ in disease progression and implicate hematopoietic cells as effectors. WT control mice and TNFRp55 $5^{-/}$mice implanted with WT bone marrow developed more tumors than WT mice transplanted with TNFRp $55^{-/-}$bone marrow. Additionally, administration of a TNF $\alpha$ antagonist to WT mice after DSS/AOM treatment attenuated colon carcinogenesis, thus demonstrating a pro-carcinogenic role for TNF $\alpha$ in this murine model of CAC.

Transcription targets of NF- $\kappa \mathrm{B}$ were shown to play a substantial role in colon tumorigenesis. IL-6 transcription results from $\mathrm{NF}-\kappa \mathrm{B}$ activation and promotes proliferation and inhibits apoptosis in intestinal epithelial cells (IECs), thus promoting colon carcinogenesis. ${ }^{26} \mathrm{DSS} / \mathrm{AOM}$ treatment of IL- $6^{-/-}$mice reduced tumor burden compared to WT mice. Additionally, with DSS-only treatment, IL- $6^{-/-}$mice showed increased inflammation and more severe colitis than WT mice. Several experiments measured both IL-6 producing cells and target cells. BrdU staining indicated that in IL- $6^{-/-}$ mice increased colitis severity is partially due to reduced proliferation of IECs. Bone marrow transplant experiments showed that hematopoietic cells play an important role in IL-6 function, while non-hematopoietic cells play a lesser role. Flow cytommetry was used to determine that during CAC development dendritic cells and macrophages are the primary IL-6-producing cells. IL-6 has a complex function in intestinal homeostasis; a protective role in colitis and a promoting role in colon carcinogenesis. ${ }^{26}$

The role of TNF $\alpha$ in intestinal inflammation is well established as evidenced by the use of anti-TNF $\alpha$ monoclonal antibody (anti-TNF $\alpha$ Mab) to treat inflammatory bowel disease (IBD). To investigate the mechanism of blocking TNF $\alpha$ on CAC development, investigators used anti-TNF $\alpha$ Mab in a DSS/AOM model. ${ }^{27}$ Specifically, to determine effects of the antibody the authors looked at TNFR1/2 signaling and NF- $\kappa \mathrm{B}$ activity in colonic epithelial cells of treated versus untreated mice. TFNR2 was upregulated in colonic epithelia and $\mathrm{NF}-\kappa \mathrm{B}$ was activated with DSS/AOM treatment. In a colitis model, one-time treatment with anti-TNF $\alpha$ Mab inhibited $\mathrm{NK}-\kappa \mathrm{B}$ activation, but did not have a significant effect on inflammation. However, repetitive treatment with anti-TNF $\alpha$ $\mathrm{Mab}$ in DSS/AOM-treated mice inhibited CAC. The authors concluded that maintenance therapy with anti-TNF $\alpha$ Mab in UC may decrease the risk of CAC.

\section{Lymphocyte-mediated immunity in CAC}

Though innate immunity is important for initiating the inflammatory response, adaptive immunity contributes to maintenance of chronic inflammatory syndromes such as IBD. Several T cells subsets are involved in IBD. Ulcerative colitis is characterized by a Th2 response of CD4+ T cells ${ }^{14}$ which contributes to CAC development. Th17 helper cells may also be involved in CAC pathogenesis, as IL-17 and IL-23 are implicated in IBD. ${ }^{28}$ The presence of other T cell subsets such as CD8+ T cells, Th1 T cells, and regulatory $\mathrm{T}$ (Tregs) cells may repress colitis-associated carcinogenesis. The Th1 response is important in anti-tumorigenic activity through activation of CD8+ T cells via IFN $\gamma$ secretion. ${ }^{14}$ Tregs inhibit CAC development through release of antiinflammatory cytokines IL-10 and TGF $\beta$. The effects of Tregs in CAC are clearly demonstrated by the spontaneous development of inflammation-associated colon cancer in IL-10-deficient mice. ${ }^{29}$ During CAC pathogenesis, T cells are implicated in the production of cytokines such as IL-6 which promotes tumor growth. ${ }^{30}$ In turn, IL-6 production promotes spontaneous proliferation of T cells. Spontaneous $\mathrm{T}$ cell proliferation and antigen-specific $\mathrm{T}$ cell proliferation are both required to induce colitis. ${ }^{31}$

The role of Th2 CD4+ T cells in UC and CAC is supported by recent research. In one study, mice genetically prone towards either a Th1 or Th2 response were treated with AOM and trinitrobenzene sulfonic acid (TNBS). In this model of CAC, IFN $\gamma$-deficient mice which produce increased IL-4 and IL-5 and develop a Th2 phenotype, showed enhanced tumor growth compared to IL-4 ${ }^{-/}$(Th1) and WT mice. ${ }^{13}$ Another study investigated the effects of subjecting IL-4Ra-deficient mice to DSS/AOM treatment. The authors observed attenuated colon carcinogenesis in IL-4Ra $\mathrm{Ra}^{-/-}$mice. These findings support the contribution of IL-4 and Th2 T cells to CAC development. ${ }^{32}$ Additionally, $60 \%$ of human colon adenocarcinomas displayed IL-4Ra expression, as did murine colon cancer. ${ }^{32}$

The Treg subset is responsible for attenuating the immune response through the production of IL-10 and TGF $\beta$. Defects in Treg development can result in inflammatory diseases such as UC. IL-10-deficient mice are used as an animal model of CAC. ${ }^{29}$ In human studies, Foxp3+ Tregs are correlated with increased survival. ${ }^{33}$ Another recent study showed that Tregs can be converted to an inflammatory Th17 phenotype by mast cells. These converted Tregs promote colon tumorigenesis rather than inhibit tumor growth. ${ }^{34}$ Interestingly, this study shows that converted Tregs express decreased levels of 
IL-10 and increased levels of IL-17, thus diminishing anti-inflammatory properties and contributing to colonic inflammation and carcinogenesis.

Research which determines the biological function of cytokines in chronic intestinal inflammation and inflammation-induced colorectal cancer provides a framework for determining the disease stage in which these proteins are involved. These clues uncover potential biomarkers for IBD and various stages of CRC. To evaluate further the utility of these proteins as biomarkers, patient cohort studies must be performed. By comparing serum protein levels in IBD and CRC patients to those in healthy controls, differential expression can be determined.

\section{Detection of serum biomarkers in murine colitis}

Animal studies may be useful to identify potential serum biomarkers. A recent study examined 23 cytokines in acute and chronic models of murine colitis. To determine the cytokine profile for both acute and recurring colitis, this study compared acute $C$. rodentium-induced colitis with Helicobacter spp.-dependent chronic colitis. Both tissue and serum cytokine levels were determined by ELISA. Multivariate modeling indicated a similar increase in chemokine and cytokine expression in the colon of animals with acute and recurring colitis. Interestingly, serum biomarkers in recurring colitis were different than those in acute colitis and included neutrophil and Th17-related factors KC, IL-12/23p40, IL-17, G-CSF and IL-6. ${ }^{15}$ These cytokines, which are expressed at higher levels in recurring versus acute colitis, may serve as important biomarkers not only for UC, but also CAC, as recurring colitis greatly increases the risk for CAC. This study demonstrated the potential for serum analysis and mathematical modeling in establishing cytokine profiles for disease. However, this study used a small sample size and murine models that may not be predictive of human disease.

\section{Proteomics and detection of cancer biomarkers}

The use of proteomics to determine differential protein expression in patient versus control serum is gaining acceptance as a method to identify disease biomarkers. Serum can be analyzed by mass spectrometry (MS) or the Rolling Circle amplification (RCA) assay. Proteins of interest can be validated using ELISA to test multiple patient cohorts. Through proteomics methods biomarker panels can be developed that are highly sensitive and specific. Serum biomarker panels are currently being developed for various cancer types.

A 2010 proteomics study identified serum proteins upregulated in CRC metastasis. ${ }^{35}$ A primary CRC cell line was compared to a metastatic cell line from the same patient to identify differential protein secretion by metastatic CRC cells in vitro; 145 proteins were differentially expressed greater than 1.5-fold. Two highly differentially expressed proteins, serum trefoil factor 3 (TFF3) and growth/differentiation factor 15 (GDF15) were analyzed in clinical samples. Their levels were significantly higher in serum from patients with lymph node metastasis. The authors conclude that TFF3 and GDF15 may be prognostic indicators of CRC lymph node metastasis. TFF3 is a small protein secreted by intestinal goblet cells. It is involved in maintaining the mucosal epithelium through restitution which entails tissue healing by the migration of epithelial cells. TTF3 is a mobility factor which can promote invasion and metastasis in cancer cells that are resistant to apoptosis. GDF15, a member of the transforming growth factor- $\beta$ (TGF- $\beta$ ) superfamily, is expressed as a propeptide and cleaved to its active secreted form. GDF15 may be involved in inhibition of growth, cell detachment and invasion, though not much is known regarding its function. ${ }^{35}$

Proteomic methods were used to determine potential biomarkers in other cancer types. A multiplex assay of six serum biomarkers is under development as a screening test for early stage ovarian cancer in high-risk patients. ${ }^{36}$ Researchers performed RCA on serum samples from ovarian cancer patients and compared results to control serum. Candidate biomarkers were further evaluated using ELISA of serum from an additional cohort. Results were repeated using a multiplex bead array which is less expensive and requires a small volume of serum thus making the test more practical for widespread studies. The final panel of six biomarkers included leptin, prolactin, osteopontin, insulin-like growth factor II, macrophage inhibitory factor and CA-125 achieved a sensitivity of $95.3 \%$ and specificity of $99.4 \%$.

Another group is using proteomics to develop a panel of biomarkers to detect and grade prostate cancer. ${ }^{37}$ Researchers used 2D difference in-gel electrophoresis (2D-DIGE) and nuclear magnetic resonance spectroscopy (NMR) to analyze serum from patients with high- and low-grade prostate cancer. MS was used to identify differentially expressed proteins. Comparison of serum samples using a panel of biomarkers was able to predict different cancer stages including metastatic versus non-metastatic prostate cancer. Patient cohort validation is necessary. 
Proteomics methods have been used to determine differential serum protein levels in disease-free versus recurring head and neck squamous cell cancer (HNSCC) patients. 2D-DIGE and MS techniques were used to analyze serum samples from patients undergoing treatment; 16 proteins were differentially expressed by disease status. ${ }^{38}$

To determine potential serum biomarkers for pancreatic cancer, Bunger et al performed a database search. They detected both individual markers and panels of biomarkers with sensitivity and specificity ranging from $77 \%-100 \%$ and $84 \%-100 \%$ respectively. Validation of markers using multiplex assays to analyze serum from patient cohorts is suggested as the next step to move toward clinical application. ${ }^{39}$

Serum proteomics analysis was used to discover differential serum biomarkers for several other types of cancer including melanoma, hepatocellular carcinoma, renal cell carcinoma, lung cancer and early stage breast cancer. ${ }^{40-44}$

Proteomics methods are useful for determining differential protein compositions of samples. Advances in these methods allow the detection of non-abundant proteins thus increasing the likelihood of discovering variations. Analysis of serum samples and secreted proteins allows the discovery of potential biomarkers for cancer diagnosis. By combining a proteomics approach with existing data on IBD and CRC it may be possible to develop accurate biomarkers for diagnosis and disease staging.

\section{Cohort studies to determine serum biomarkers in CRC}

Cohort studies are a valuable method of validating serum proteins as potential CRC biomarkers. Development of biomarker panels may be useful for screening and monitoring patients for CAC and SCRC progression. Patient cohort and animal studies identified serum biomarkers with equivocal results. Most patient cohort studies compare CRC development in patients without preexisting conditions to average risk controls (without IBD). Some studies include patients and controls with additional confounders such as IBD, obesity and smoking. Many studies are limited in breadth with only a few cytokines examined, or use a limited population wherein significant differences are not detected. Due to the complexity of cytokine expression in UC, CAC and SCRC it may be necessary to examine extensive arrays of serum biomarkers using large patient populations to detect significant differences in serum cytokine levels and for cohort studies to ultimately have diagnostic and prognostic value.
TNF $\alpha$ is an acute phase pro-inflammatory cytokine that activates the transcription factor NF- $\mathrm{KB}$ thus promoting the expression of several downstream mediators of inflammation. A patient cohort study indicated that TNF $\alpha$ expression is increased in $\mathrm{UC}, \mathrm{CD}$ and $\mathrm{CRC}$ compared to healthy controls. ${ }^{45}$ Also, TNF $\alpha$ expression is higher in $\mathrm{CRC}$ than in $\mathrm{UC}$ patients. This study, by Szkaradkiewicz et al, used a small sample number and analyzed serum collected from patients at the time of diagnosis. ${ }^{45} \mathrm{~A}$ study by Kim et al indicates that TNF $\alpha$ is associated with increased risk of colorectal adenoma. ${ }^{46} \mathrm{This}$ study used a predominately obese population to determine if obesity is associated with inflammation and CRC. It was determined that obesity correlates with increased levels of inflammatory cytokines and it is a risk factor in CRC development. A recent study also indicates that $\mathrm{TNF} \alpha$ correlates with increased risk of CRC. ${ }^{47}$ This study measured an alternative marker for TNF $\alpha$, soluble TNF-receptor-2 (sTNFR-2), because it is more stable in stored frozen samples. This study by Chan et al analyzed data from the Nurses' Health Study which is a well-documented case-control study sampled from a large population. This study required a baseline serum collection and had a 15 -year follow-up. ${ }^{47}$ Another alternative marker for TNF $\alpha$, soluble TNF-receptor-1 (sTNFR-1) was found to correlate with tumor grade and invasion ${ }^{48}$ in a study that used a small control population and serum samples were collected at the time of diagnosis. The Bobe et al and Il'yasova et al studies found no correlation of TNF $\alpha$ with CRC occurrence. . $^{5,49}$

Mediators of inflammation downstream from TNF $\alpha$ have also been investigated. TNF $\alpha$ signaling activates the transcription factor NF- $\kappa$ B resulting in expression of IL-6. Many studies investigated the correlation of pro-inflammatory cytokine IL-6 with CRC risk. Several studies, including those by Kim et al and Bobe et al, found that increased levels of IL-6 correlate with increased risk for $\mathrm{CRC}^{5,45,46,50-52}$ while other studies have found no association. ${ }^{47,53,54}$ Il'yasova et al investigated patients 70-79 years of age. Serum was collected initially and follow-up of patients was 5.5 years. They found that increased IL-6 correlated with CRC occurrence and the association was greater with CRC mortality. ${ }^{5}$ Malicki et al measured cytokine levels in a small cohort of CRC patients and compared the results to healthy controls. They found that IL-6 levels were higher in CRC patients. Interestingly, they found that serum IL-6 levels decreased after surgical removal of tumors. ${ }^{50}$ Knupfer et al analyzed previously published results (a total of 31 articles) to determine correlations between IL- 6 and CRC. They found that increased IL- 6 is 
positively associated with CRC and that IL- 6 concentration is associated with increased tumor size, stage, metastasis, and decreased survival. The authors conclude that IL- 6 may be a valuable prognostic indicator for CRC. ${ }^{52}$ Furthermore, the Szkaradkiewicz study found that IL-6 serum concentration is higher in UC and CD patients. ${ }^{45}$ No correlation was found by the Ognjanovic group. Serum was collected from adenoma patients at the time of diagnosis and compared to healthy controls. The sample population was multiethnic and baseline cytokine concentration varied according to ethnicity. ${ }^{53}$ Therefore, variables within cases and controls may have prevented detection of a correlation.

C-reactive protein (CRP) is an inflammatory protein that is produced by the liver in response to IL-6. CRP has been investigated for potential use as a predictive tool in many cancer types including CRC. Several studies found increased CRP to be associated with higher CRC risk. ${ }^{54-57}$ Kwon et a ${ }^{54}$ collected data from CRC patients at the time of diagnosis and found increased CRP levels in comparison to healthy controls. Studies by Gunter et al,${ }^{55}$ Erlinger et al, ${ }^{56}$ and Otani et $\mathrm{al}^{57}$ were case-control studies with serum collection at the baseline and 5 to 11 year follow-up periods. The Gunter et al study cohort was male smokers only. The Erlinger et al and Otani et al studies included men and women. The studies found that increased CRP levels increase risk of CRC development. The Aleksandrova et al case-control study included both men and women and concluded that increased CRC risk associated with elevated CRP is found only in men. ${ }^{58} \mathrm{~A}$ previous study by Zhang et al found no association in women..$^{59}$ The Zhang study was an intervention trial using aspirin, but analysis was not different between the treatment groups. A study by Kim et al found that increased CRP levels correlate with colorectal adenoma and obesity. ${ }^{46} \mathrm{Il}$ 'yasova et al found a correlation with increased CRP and cancer death. ${ }^{5}$ A meta-analysis of several cohort studies detected a low association of increased CRP with CRC risk. Heikkila et al pooled data from 10 studies. The lack of significance may have been caused by confounders such as age, smoking, socioeconomic status, obesity and activity level. ${ }^{60}$ Several studies found no association. . $7,53,61^{2}$

The NLR inflammasome product IL- $1 \beta$ is a primary stimulator of innate immunity. Though some research supports a protective role for IL-1 $\beta$ in CRC, Szkaradkiewicz found a correlation between increased IL- $1 \beta$ and UC, CD and CRC. Patients with UC and CRC had higher levels than those with $\mathrm{CD}^{45}$ Bobe et al detected an association with IL-1 $\beta$ and advanced colon adenomas. ${ }^{49}$ The Bobe et al study was a nutritional intervention trial to determine if colorectal adenoma recurrence can be decreased by increased fruit, vegetable and fiber intake and decreased fat intake. All participants had previous adenomas. The study recorded changes in diet, serum cytokine levels and adenoma recurrence over 3 years. They found that a decrease in IL- $1 \beta$ was associated with a decreased risk of advanced CRC.

The cytokine IL-23 induces inflammation through the activation of IL-17-producing T cells and myeloid cells. This heterodimeric cytokine is composed of subunits $\mathrm{p} 40$ and $\mathrm{p} 19$. The inflammatory cytokine IL-12 induces the production of IFN $\gamma$ and promotes Th1 cell differentialtion. IL-12 is also a heterodimer composed of $\mathrm{p} 40$ and $\mathrm{p} 35$ subunits. The $\mathrm{p} 40$ subunit is common to both IL-23 and IL-12 therefore studies that detect p40 may be determining levels of both IL-23 and IL-12. Studies have associated increased IL-23 levels with CRC risk. ${ }^{62,63}$ Miteva et al analyzed levels of the p40 subunit and found the highest IL-23/IL-12 serum concentration occurs in early stage CRC. ${ }^{63} \mathrm{~A}$ small patient cohort study by Ljujic et al examined CRC patient and healthy control serum for p19 concentration and found that IL-23 levels are higher in CRC patients. ${ }^{62}$ Furthermore, Bobe et al found that increased IL-12 is associated with advanced adenoma recurrence. ${ }^{49}$

Th17 differentiation is induced by IL-23 and results in increased production of the inflammatory cytokine IL-17. A study by Radosavljevic et al indicates that IL-17 levels are higher in CRC patients compared to normal controls. ${ }^{64}$ This study used the same patient cohort as the Ljujic study. The authors concluded that IL-17 may be a useful tool for CRC diagnosis.

The chemokine IL-8 induces the migration of leukocytes including neutrophil and macrophage to sites of infection. Szkaradkiewicz et al detected increased levels of IL-8 in the serum of UC, CD and CRC patients. Furthermore, CRC patients had significantly higher levels of serum IL-8 than UC patients. ${ }^{45}$ Other studies also correlated increased levels of IL-8 with CRC. ${ }^{45,48,50}$ Kaminska et al found that IL-8 significantly increases with advanced clinical stage and invasion. ${ }^{48}$ Furthermore, CRC patients had significantly higher levels of serum IL- 8 than UC patients. ${ }^{45}$ The study by Bobe et al found no association. ${ }^{49}$

IL-2 induces the proliferation of thymocytes including cytotoxic T cells, Th1, Th2, Th17 and Treg subsets. The study by Bobe et al indicated that decreased levels of IL-2 are associated with an increased risk of adenoma recurrence. ${ }^{49}$ 
Table I Cytokines associated with inflammatory bowel disease (IBD), ulcerative colitis (UC), colorectal cancer (CRC) and colitisassociated cancer (CAC)

\begin{tabular}{|c|c|c|c|}
\hline Cytokine & Function & Role in disease & References \\
\hline \multirow[t]{4}{*}{ IL-I $\beta$} & Pro-inflammatory & Primary response to IEC bacterial infiltration & 13 \\
\hline & & Protective against tumor growth in a mouse model & 15 \\
\hline & & Associated with advanced adenoma & 38 \\
\hline & & Associated with UC, CD and CRC & 45 \\
\hline \multirow[t]{3}{*}{ IL-2 } & $\mathrm{T}$ cell response & T cells contribute to chronic IBD & 7 \\
\hline & Th2 response & Decreased levels associated with adenoma recurrence & 49 \\
\hline & & Associated with CD & 8 \\
\hline \multirow[t]{2}{*}{ IL-4 } & Th2 response & Associated with UC & 7 \\
\hline & & Promotes tumor growth in mouse model of CRC & \\
\hline IL-5 & Th2 response & Characteristic of UC & 8 \\
\hline \multirow[t]{2}{*}{ IL-6 } & Pro-inflammatory & Associated with increased CRC occurrence, and mortality & $45,46,50-52$ \\
\hline & & Associated with UC and CD & 8 \\
\hline IL-8 (CXCL8) & Neutrophil chemoattractant & Associated with UC, CD and CRC & $45,48,50$ \\
\hline \multirow[t]{3}{*}{ IL-10 } & Anti-inflammatory & Lower levels associated with CRC and higher levels with UC & 45 \\
\hline & $\mathrm{T}$ reg response & Reduced levels increase tumor growth in mice & 8,23 \\
\hline & & Increased levels associated with decreased risk of advanced adenoma & 49 \\
\hline IL-I I & ThI response & Survival and growth & I \\
\hline \multirow[t]{3}{*}{ IL-I2 (p35/p40) } & Pro-inflammatory & Characteristic of UC & 8 \\
\hline & ThI 7 response & Predictive for chronic colitis in comparison to acute colitis & 9 \\
\hline & & Increased in Stage I CRC patients & 63 \\
\hline \multirow[t]{4}{*}{ IL-17 } & ThI7 response & Predictive for chronic colitis in comparison to acute colitis & 9 \\
\hline & Pro-inflammatory & Associated with murine colitis & 8 \\
\hline & & Elevated in CD patients & 41 \\
\hline & & Increased level in CRC patients & 64 \\
\hline \multirow[t]{2}{*}{ IL-18 } & Pro-inflammatory & Primary response to bacterial infiltration in IBD & 16 \\
\hline & & Protective against tumor growth in mouse model & 15 \\
\hline IL-22 & Innate immunity & Survival & 1 \\
\hline \multirow[t]{2}{*}{ IL-23 (p40/p|9) } & Pro-inflammatory & Involved in murine colitis and CAC & 56 \\
\hline & & Associated with increased risk of CRC & 62 \\
\hline \multirow[t]{4}{*}{$\mathrm{TNF} \alpha$} & Pro-inflammatory & Chronic expression present in IBD & 39 \\
\hline & & Higher concentration associated with adenoma & 21 \\
\hline & & Involved in murine colitis and CRC & 1,30 \\
\hline & & Associated with increased CRC occurrence & $45-48$ \\
\hline \multirow[t]{2}{*}{$\mathrm{IFN} \gamma$} & ThI response & Associated with improved prognosis of CRC & 7 \\
\hline & & Characteristic of CD & I \\
\hline KC (CXCLI) & Neutrophil migration & Predictive for chronic colitis in comparison to acute colitis & 9 \\
\hline G-CSF & Neutrophil response & Predictive for chronic colitis in comparison to acute colitis & 9 \\
\hline \multirow[t]{2}{*}{ TGF $\beta$} & Anti-inflammatory & Characteristic of UC & 8 \\
\hline & Th2 response & EMT, CRC promotion and invasion & I \\
\hline CRP & Pro-inflammatory & Associated with adenoma and CRC & $54-58$ \\
\hline
\end{tabular}

The Treg subset is responsible for attenuating the immune response in part through the production of anti-inflammatory cytokine IL-10. Szkaradkiewicz et al compared circulating levels of IL-10 in healthy individuals to those in patients with UC, CD or CRC. They detected increased levels of IL-10 in UC patients but not in CD or CRC patients compared to control cases. ${ }^{45}$ This study may implicate the loss of systemic IL-10 in CRC development. Bobe et al found that increased IL-10 concentration correlated with increased risk of advanced adenoma recurrence. ${ }^{49}$ These studies may have produced different results because the first study utilized a patient cohort with an initial diagnosis of CRC while the second study detected CRC recurrence.
Macrophage migration inhibitory factor (MIF) is an inflammatory protein involved in many cellular processes including differentiation and proliferation. MIF also plays a role in angiogenesis, tumor promotion and metastasis. Recent studies indicate that MIF is significantly upregulated in $\mathrm{CRC}^{65}$ and metastases. ${ }^{66}$ Analysis of serum from $128 \mathrm{CRC}$ patients with various stages of cancer compared to 53 healthy controls indicated that MIF is upregulated at all stages of CRC. Interestingly, this study found that MIF demonstrated the highest sensitivity out of 9 candidate markers in early cancer detection at $71.4 \%$, with a specificity of $90.6 \%$. Another small study that categorized CRC patients by presence of metastasis found that serum MIF levels significantly correlated with 
$\mathrm{CRC}$ and that the correlation was greater in patients with metastasis. These studies indicate a potential role for MIF as a biomarker for CRC though studies need to be done in a larger population to verify the correlation.

Serum hepatocyte growth factor (SHGF) is involved in proliferation and angiogenesis and was detected in the serum of cancer patients including those with CRC. ${ }^{67}$ In a 2009 study, SHGF was significantly elevated in CRC patients compared to controls. Additionally, SHGF was significantly increased in advanced stages (II and III) which include patients with large tumors and metastasis. HGF levels correlated with poor survival and may serve as a prognostic indicator.

Other cytokines were found to have no correlation with CRC and many potential cytokines have not yet been investigated. Additional cytokines analyzed by Bobe et al and found not to correlate with CRC risk include INF $\gamma$ and $\mathrm{GMCSF}^{49}{ }^{4}$ The Bobe et al study was conducted as a nutritional intervention trial and may not be conclusive for defining cytokine profiles in CRC.

Many cytokines were evaluated as potential CRC biomarkers and several factors that have been implicated in CRC have not yet been investigated. In addition, most studies use patient cohorts with CRC and no previous IBD diagnosis. These studies are useful for developing diagnostic and prognostic tools for the average-risk population against CRC. Studies that analyze the differential cytokine expression in UC compared to CAC are lacking and may be helpful in developing diagnostic tools for monitoring UC patients. Many studies involved small numbers of patients, some with high variability between cases and controls, and many studies only measured a limited number of factors. To detect significant differences in serum protein levels it may be necessary to perform large case-control studies with analysis of extensive panels of biomarkers. Determining differential expression of multiple serum biomarkers in various stages of disease may help in the development of diagnostic and prognostic assays with high sensitivity and specificity. Existing data suggest potential biomarkers for CRC. Also, the use of animal models of IBD and CRC may help to discover additional mediators of disease. Furthermore, emerging proteomic methods are capable of thorough analysis of serum proteins and may be used to determine differential expression of factors in CRC patient samples. Continuing research using both animal models and proteomics followed by validation with cohort studies may determine the appropriate combinations of serum biomarkers for multivariate models of disease status and stage.

\section{Conclusion}

Many cytokines and serum proteins are involved in IBD and CRC (Table 1). These factors regulate diverse functions including initiation of the innate immune response, stimulation of cell-mediated adaptive immunity, homeostasis, cell migration, survival and anti-inflammatory responses. Several cytokines have a well-established role in IBD such as TNF $\alpha$, IL-1 $\beta$, IL-2, IL-4, IL-5, IL-6, IL-10, IL-12, IL-17, IL-18, KC and G-CSF. Inflammatory cytokines, particularly those promoting a Th2 and Th17 response, are associated with UC. Inflammatory cytokines that initiate a Th1 response, such as IFN $\gamma$ are associated with CD. Many cytokines are associated with colon neoplasia and CRC stage including TNF $\alpha$, IL-1 $\beta$, IL-6, IL-8, IL-12/IL-23 and IL-17. Roles for other cytokines and serum proteins are still being established. Not only do these serum proteins have important roles in disease pathogenesis, they also have potential as biomarkers for chronic inflammation, colon carcinogenesis, invasion and metastasis. Due to the minimal invasiveness of serum testing in patients and the cost-effectiveness of multiplex assays, discovery of serum biomarkers could lead to the development of an efficient method of diagnosing CAC and SCRC. Defining serum biomarker profiles specific to various stages of CRC may reduce mortality by improving diagnosis of early-stage disease and alerting healthcare providers to disease progression.

\section{Acknowledgments}

Grant support: Office of Research and Development, Medical Research Service, Department of Veterans Affairs (J-P. Raufman), and NIH grants CA107345 and CA120407 (J-P. Raufman).

\section{Disclosure}

The authors report no conflicts of interest in this work.

\section{References}

1. Terzic J, Grivennikov S, Karin E, Karin M. Inflammation and colon cancer. Gastroenterology. 2010;138(6):2101-2114.

2. Laukoetter MG, Mennigen R, Hannig CM, et al. intestinal cancer risk in Crohn's disease: a meta-analysis. J Gastrointest Surg. 2010; 15(4):576-583.

3. Triantafillidis JK, Nasioulas G, Kosmidis PA. Colorectal cancer and inflammatory bowel disease: epidemiology, risk factors, mechanisms of carcinogenesis and prevention strategies. Anticancer Res. 2009; 29(7):2727-2737.

4. Clevers H. At the crossroads of inflammation and cancer. Cell. 2004; 118(6):671-674.

5. Il'yasova D, Colbert LH, Harris TB, et al. Circulating levels of inflammatory markers and cancer risk in the health aging and body composition cohort. Cancer Epidemiol Biomarkers Prev. 2005;14(10): 2413-2418.

6. Link KH, Sagban TA, Morschel M, et al. Colon cancer: survival after curative surgery. Langenbecks Arch Surg. 2005;390(2):83-93.

7. Baxter NN, Goldwasser MA, Paszat LF, Saskin R, Urbach DR, Rabeneck L. Association of colonoscopy and death from colorecta cancer. Ann Intern Med. 2009;150(1):1-8. 
8. Lieberman D. Colon cancer screening and surveillance controversies. Curr Opin Gastroenterol. 2009;25(5):422-427.

9. Van Dam L, Kuipers EJ, van Leerdam ME. Performance improvements of stool-based screening tests. Best Pract Res Clin Gastroenterol. 2010; 24(4):479-492.

10. Lund EK, Belshaw NJ, Elliott GO, Johnson IT. Recent advances in understanding the role of diet and obesity in the development of colorectal cancer. Proc Nutr Soc. 2011:1-11.

11. Kulaylat MN, Dayton MT. Ulcerative colitis and cancer. J Surg Oncol. 2010;101(8):706-712.

12. Coussens LM, Werb Z. Inflammation and cancer. Nature. 2002; 420(6917):860-867.

13. Osawa E, Nakajima A, Fujisawa T, et al. Predominant T helper type 2-inflammatory responses promote murine colon cancers. Int J Cancer. 2006;118(9):2232-2236.

14. Waldner MJ, Neurath MF. Colitis-associated cancer: the role of T cells in tumor development. Semin Immunopathol. 2009;31(2):249-256.

15. McBee ME, Zeng Y, Parry N, Nagler CR, Tannenbaum SR, Schauer DB Multivariate modeling identifies neutrophil- and Th17-related factors as differential serum biomarkers of chronic murine colitis. PLoS One. 2010;5(10):e13277

16. Wang CS, Sun CF. C-reactive protein and malignancy: clinicopathological association and therapeutic implication. Chang Gung Med J. 2009;32(5):471-482.

17. Yan Y, Kolachala V, Dalmasso G, et al. Temporal and spatial analysis of clinical and molecular parameters in dextran sodium sulfate induced colitis. PLoS ONE. 2009;4(6):e6073.

18. Schroder K, Zhou R, Tschopp J. The NLRP3 inflammasome: a sensor for metabolic danger? Science. 2010;327(5963):296-300.

19. Barber MD, Powell JJ, Lynch SF, Fearon KC, Ross JA. A polymorphism of the interleukin-1 beta gene influences survival in pancreatic cancer. Br J Cancer. 2000;83(11):1443-1447.

20. Allen IC, TeKippe EM, Woodford RM, et al. The NLRP3 inflammasome functions as a negative regulator of tumorigenesis during colitis-associated cancer. J Exp Med. 2010;207(5):1045-1056.

21. Zaki MH, Boyd KL, Vogel P, Kastan MB, Lamkanfi M, Kanneganti TD. The NLRP3 inflammasome protects against loss of epithelial integrity and mortality during experimental colitis. Immunity. 2010;32(3):379-391.

22. Xiao H, Yin W, Khan MA, et al. Loss of single immunoglobulin interlukin-1 receptor-related molecule leads to enhanced colonic polyposis in Apc(min) mice. Gastroenterology. 2010;139(2):574-585.

23. Dupaul-Chicoine J, Yeretssian G, Doiron K, et al. Control of intestinal homeostasis, colitis, and colitis-associated colorectal cancer by the inflammatory caspases. Immunity. 2010;32(3):367-378.

24. Salcedo R, Worschech A, Cardone M, et al. MyD88-mediated signaling prevents development of adenocarcinomas of the colon: role of interleukin 18. J Exp Med. 2010;207(8):1625-1636.

25. Popivanova BK, Kitamura K, Wu Y, et al. Blocking TNF-alpha in mice reduces colorectal carcinogenesis associated with chronic colitis. J Clin Invest. 2008;118(2):560-570.

26. Grivennikov S, Karin E, Terzic J, et al. IL-6 and Stat3 are required for survival of intestinal epithelial cells and development of colitisassociated cancer. Cancer Cell. 2009;15(2):103-113.

27. Onizawa M, Nagaishi T, Kanai T, et al. Signaling pathway via TNFalpha/NF-kappaB in intestinal epithelial cells may be directly involved in colitis-associated carcinogenesis. Am J Physiol Gastrointest Liver Physiol. 2009;296(4):G850-G859.

28. Neurath MF. IL-23: a master regulator in Crohn disease. Nat Med. 2007;13(1):26-28.

29. Berg DJ, Davidson N, Kuhn R, et al. Enterocolitis and colon cancer in interleukin-10-deficient mice are associated with aberrant cytokine production and CD4(+) TH1-like responses. J Clin Invest. 1996;98(4):1010-1020.

30. Becker C, Fantini MC, Wirtz S, et al. IL-6 signaling promotes tumor growth in colorectal cancer. Cell Cycle. 2005;4(2):217-220.
31. Feng T, Wang L, Schoeb TR, Elson CO, Cong Y. Microbiota innate stimulation is a prerequisite for $\mathrm{T}$ cell spontaneous proliferation and induction of experimental colitis. J Exp Med. 2010;207(6):1321-1332.

32. Koller FL, Hwang DG, Dozier EA, Fingleton B. Epithelial interleukin-4 receptor expression promotes colon tumor growth. Carcinogenesis. 2010;31(6):1010-1017.

33. Salama P, Phillips M, Grieu F, et al. Tumor-infiltrating FOXP3+ $\mathrm{T}$ regulatory cells show strong prognostic significance in colorectal cancer. J Clin Oncol. 2009;27(2):186-192.

34. Blatner NR, Bonertz A, Beckhove P, et al. In colorectal cancer mast cells contribute to systemic regulatory T-cell dysfunction. Proc Natl Acad Sci U S A. 2010;107(14):6430-6435.

35. Xue H, Lu B, Zhang J, et al. Identification of serum biomarkers for colorectal cancer metastasis using a differential secretome approach. J Proteome Res. 2010;9(1):545-555.

36. Kim K, Visintin I, Alvero AB, Mor G. Development and validation of a protein-based signature for the detection of ovarian cancer. Clin Lab Med. 2009;29(1):47-55.

37. Fan Y, Murphy TB, Byrne JC, Brennan L, Fitzpatrick JM, Watson RW. Applying Random Forests to identify biomarker panels in serum 2D-DIGE data for the detection and staging of prostate cancer. J Proteome Res. 2011;10(3):1361-1373.

38. Gourin CG, Zhi W, Adam BL. Proteomic identification of serum biomarkers for head and neck cancer surveillance. Laryngoscope. 2009;119(7):1291-1302.

39. Bunger S, Laubert T, Roblick UJ, Habermann JK. Serum biomarkers for improved diagnostic of pancreatic cancer: a current overview. J Cancer Res Clin Oncol. 2011;137(3):375-389.

40. Greco M, Mitri MD, Chiriaco F, Leo G, Brienza E, Maffia M. Serum proteomic profile of cutaneous malignant melanoma and relation to cancer progression: association to tumor derived alpha-N-acetylgalactosaminidase activity. Cancer Lett. 2009;283(2):222-229.

41. Zinkin NT, Grall F, Bhaskar K, et al. Serum proteomics and biomarkers in hepatocellular carcinoma and chronic liver disease. Clin Cancer Res. 2008;14(2):470-477.

42. Sarkissian G, Fergelot P, Lamy PJ, et al. Identification of pro-MMP-7 as a serum marker for renal cell carcinoma by use of proteomic analysis. Clin Chem. 2008;54(3):574-581.

43. Yildiz PB, Shyr Y, Rahman JS, et al. Diagnostic accuracy of MALDI mass spectrometric analysis of unfractionated serum in lung cancer. J Thorac Oncol. 2007;2(10):893-901.

44. Belluco C, Petricoin EF, Mammano E, et al. Serum proteomic analysis identifies a highly sensitive and specific discriminatory pattern in stage 1 breast cancer. Ann Surg Oncol. 2007;14(9):2470-2476.

45. Szkaradkiewicz A, Marciniak R, Chudzicka-Strugala I, et al. Proinflammatory cytokines and IL-10 in inflammatory bowel disease and colorectal cancer patients. Arch Immunol Ther Exp (Warsz). 2009; 57(4):291-294

46. Kim S, Keku TO, Martin C, et al. Circulating levels of inflammatory cytokines and risk of colorectal adenomas. Cancer Res. 2008;68(1): 323-328.

47. Chan AT, Ogino S, Giovannucci EL, Fuchs CS. Inflammatory markers are associated with risk of colorectal cancer and chemopreventive response to anti-inflammatory drugs. Gastroenterology. 2011;140(3): 799-808.

48. Kaminska J, Nowacki MP, Kowalska M, et al. Clinical significance of serum cytokine measurements in untreated colorectal cancer patients: soluble tumor necrosis factor receptor type I-an independent prognostic factor. Tumour Biol. 2005;26(4):186-194.

49. Bobe G, Murphy G, Albert PS, et al. Serum cytokine concentrations, flavonol intake and colorectal adenoma recurrence in the Polyp Prevention Trial. Br J Cancer. 2010;103(9):1453-1461.

50. Malicki S, Winiarski M, Matlok M, Kostarczyk W, Guzdek A, Konturek PC. IL-6 and IL-8 responses of colorectal cancer in vivo and in vitro cancer cells subjected to simvastatin. J Physiol Pharmacol. 2009;60(4):141-146. 
51. Bobe G, Albert PS, Sansbury LB, et al. Interleukin-6 as a potential indicator for prevention of high-risk adenoma recurrence by dietary flavonols in the polyp prevention trial. Cancer Prev Res (Phila). 2010; 3(6):764-775.

52. Knupfer H, Preiss R. Serum interleukin-6 levels in colorectal cancer patients-a summary of published results. Int J Colorectal Dis. 2010; 25(2):135-140.

53. Ognjanovic S, Yamamoto J, Saltzman B, et al. Serum CRP and IL-6, genetic variants and risk of colorectal adenoma in a multiethnic population. Cancer Causes Control. 2010;21(7):1131-1138.

54. Kwon KA, Kim SH, Oh SY, et al. Clinical significance of preoperative serum vascular endothelial growth factor, interleukin-6, and C-reactive protein level in colorectal cancer. BMC Cancer. 2010;10:203.

55. Gunter MJ, Stolzenberg-Solomon R, Cross AJ, et al. A prospective study of serum C-reactive protein and colorectal cancer risk in men. Cancer Res. 2006;66(4):2483-2487.

56. Erlinger TP, Platz EA, Rifai N, Helzlsouer KJ. C-reactive protein and the risk of incident colorectal cancer. JAMA. 2004;291(5):585-590.

57. Otani T, Iwasaki M, Sasazuki S, Inoue M, Tsugane S. Plasma C-reactive protein and risk of colorectal cancer in a nested case-control study: Japan Public Health Center-based prospective study. Cancer Epidemiol Biomarkers Prev. 2006;15(4):690-695.

58. Aleksandrova K, Jenab M, Boeing H, et al. Circulating C-reactive protein concentrations and risks of colon and rectal cancer: a nested case-control study within the European Prospective Investigation into Cancer and Nutrition. Am J Epidemiol. 2010;172(4):407-418.

59. Zhang SM, Buring JE, Lee IM, Cook NR, Ridker PM. C-reactive protein levels are not associated with increased risk for colorectal cancer in women. Ann Intern Med. 2005;142(6):425-432.
60. Heikkila K, Harris R, Lowe G, et al. Associations of circulating $\mathrm{C}$-reactive protein and interleukin-6 with cancer risk: findings from two prospective cohorts and a meta-analysis. Cancer Causes Control. 2009;20(1):15-26.

61. Ito Y, Suzuki K, Tamakoshi K, et al. Colorectal cancer and serum C-reactive protein levels: a case-control study nested in the JACC Study. J Epidemiol. 2005;15 Supp1 2:S185-S189.

62. Ljujic B, Radosavljevic G, Jovanovic I, et al. Elevated serum level of IL-23 correlates with expression of VEGF in human colorectal carcinoma. Arch Med Res. 2010;41(3):182-189.

63. Miteva L, Stanilov N, Deliysky T, Mintchev N, Stanilova S. Association of polymorphisms in regulatory regions of interleukin-12p40 gene and cytokine serum level with colorectal cancer. Cancer Invest. 2009; 27(9):924-931.

64. Radosavljevic G, Ljujic B, Jovanovic I, et al. Interleukin-17 may be a valuable serum tumor marker in patients with colorectal carcinoma. Neoplasma. 2010;57(2):135-144.

65. Lee H, Rhee H, Kang HJ, et al. Macrophage migration inhibitory factor may be used as an early diagnostic marker in colorectal carcinomas. Am J Clin Pathol. 2008;129(5):772-779.

66. He XX, Chen K, Yang J, et al. Macrophage migration inhibitory factor promotes colorectal cancer. Mol Med. 2009;15(1-2):1-10.

67. Toiyama Y, Miki C, Inoue Y, Okugawa Y, Tanaka K, Kusunoki M. Serum hepatocyte growth factor as a prognostic marker for stage II or III colorectal cancer patients. Int J Cancer. 2009;125(7):1657-1662.
Gastrointestinal Cancer: Targets and Therapy

\section{Publish your work in this journal}

Gastrointestinal Cancer: Targets and Therapy is an international, peer-reviewed, open access journal focusing on gastro-intestinal cancer research, identification of therapeutic targets and the optimal use of preventative and integrated treatment interventions to achieve improved outcomes, enhanced survival and quality of life for the

\section{Dovepress}

cancer patient. The manuscript management system is completely online and includes a very quick and fair peer-review system. Visit http://www.dovepress.com/testimonials.php to read real quotes from published authors. 